Gestionar: revista de empresa y gobierno

Vol. 1 Núm. 1 (2021)

ISSN: $2810-8264$ ISSN-L: 2810-823X

Editada por: Instituto Universitario de Innovación Ciencia y Tecnología Inudi Perú

\title{
Habilidades psicosociales y su influencia en niveles de riesgo de estudiantes de secundaria
}

\author{
Psychosocial skills and their influence on risk levels of high school students
}

Habilidades psicossociais e sua influência nos níveis de risco de estudantes do ensino médio

Lila Salas ${ }^{1}$

Universidad César Vallejo, Perú

Fany Montaño

Universidad César Vallejo, Perú

Carlos Yengle

Universidad César Vallejo, Perú

DOI: https://doi.org/10.35622/j.rg.2021.01.002

Recibido 02/12/2020/ Aceptado 30/01/2021

RESUMEN. El objetivo fue determinar la influencia de las habilidades psicosociales en el nivel de riesgo de estudiantes de nivel secundario distrito de Hunter Arequipa - 2018. Se utilizó el enfoque cuantitativo, de tipo descriptivo correlacional, de nivel explicativo y diseño no experimental. La población y muestra consta de 265 estudiantes de dos instituciones educativas del nivel secundario del sector público del distrito de Hunter. Los datos fueron procesados en los programas Excel y SPSS V22. Para la primera variable de habilidades psicosociales se encontró que un $56.60 \%$ se ubica en el nivel alto, un $30.20 \%$ se ubica en nivel bajo y un $13.2 \%$ en nivel regular. La segunda variable nivel de riesgo indica que un $35.50 \%$ se ubica en el nivel bajo, un $34.30 \%$ se ubica en nivel regular y un $30.20 \%$ en nivel alto. Se ha logrado determinar que la correlación entre las dos variables explica un $81 \%$ de la varianza de la variable dependiente, con R2 corregido de 0,814 . Además, el error típico de los residuos ha disminuido, lo que indica una pequeña mejora en el ajuste.

PALABRAS CLAVE: habilidad psicosocial, riesgo individual, riesgo social, riesgo familiar.

\begin{abstract}
The objective was to determine the influence of psychosocial skills on the level of risk of high school students in the Hunter Arequipa district - 2018. The quantitative approach, descriptive-correlational, explanatory level, and nonexperimental design were used. The population and sample consisted of 265 students from two Hunter District public sector secondary level educational institutions. The data were processed in the Excel and SPSS V22 programs. For the first variable of psychosocial skills, it was found that $56.60 \%$ are located at the high level, $30.20 \%$ are
\end{abstract}

${ }^{1}$ Correspondencia: ilujanom@est.unap.edu.pe 
located at the low level, and 13.2\% are at the average level. The second variable level of risk indicates that $35.50 \%$ are at the low level, $34.30 \%$ are at the intermediate level, and $30.20 \%$ are at the high level. It has been determined that the correlation between the two variables explains $81 \%$ of the variance of the dependent variable, with a corrected $\mathrm{R} 2$ of 0.814 . In addition, the standard error of the residuals has decreased, indicating a slight improvement in the fit.

KEYWORDS: psychosocial ability, individual risk, social risk, family risk.

RESUMO. O objetivo foi determinar a influência das habilidades psicossociais no nível de risco de estudantes do ensino médio no distrito de Hunter Arequipa - 2018. Utilizouse a abordagem quantitativa, descritiva-correlacional, nivel explicativo e delineamento não experimental. A população e a amostra consistem em 265 alunos de duas instituições de ensino médio do setor público no distrito de Hunter. Os dados foram processados nos programas Excel e SPSS V22. Para a primeira variável de habilidades psicossociais, verificou-se que $56,60 \%$ estão no nível alto, 30,20\% no nível baixo e $13,2 \%$ no nível regular. A segunda variável nível de risco indica que 35,50\% estão no nível baixo, 34,30\% no nível regular e 30,20\% no nivel alto. Foi possivel determinar que a correlação entre as duas variáveis explica $81 \%$ da variância da variável dependente, com um R2 corrigido de 0,814 . Além disso, o erro padrão dos resíduos diminuiu, indicando uma pequena melhora no ajuste.

PALABRAS CLAVE: habilidade psicossocial, risco individual, risco social, risco familiar.

\section{INTRODUCCIÓN}

A nivel mundial, según el Fondo de las Naciones Unidas para la Infancia - UNICEF, la tasa de embarazos de jóvenes en todo el mundo en las cercanías de 15 y 19 años es de 15 millones que conciben una descendencia. Además, el 37\% de esta exhiben una alta utilización de licor, donde el período normal de inicio es de 13 años. En cuanto a la utilización del tabaco todos los días, aproximadamente 3,000 menores intentan fumarse un cigarrillo, mientras que 700 menores progresan y se convierten en fumadores. De manera similar se estima que 150 millones de menores devoran tabaco y que se está expandiendo.

A nivel nacional, el Perú muestra alrededor de 5 millones de jóvenes entre 10 y 19 años. Por otra parte, la Encuesta demográfica y de salud familiar ENDES (2014) aparece en el supuesto de que el 26,7\% de los jóvenes de 15 a 19 años de edad son ahora madres y el 4,6\% están embarazadas. El año 2017 se registró en la zona rural un $24.7 \%$ y urbana un $12.2 \%$. 
Bermejo y Fernández (2010) realizaron la investigación titulada: "Habilidades sociales y resolución de conflictos en centros docentes de Andalucía", donde encuentran que la mayoría del profesorado tiene una valoración negativa del clima que existe en los centros educativos, resaltan la influencia que tienen los medios de comunicación en la comunidad educativa.

Por su parte Verde, R. (2015) en su tesis: aplicación de estrategias de habilidades sociales para optimizar las relaciones humanas en los estudiantes del segundo grado de nivel secundario de la I.E. Padre Pérez de Guereñu - Paucarpata - Arequipa encuentra que, si los estudiantes no saben desarrollar habilidades psicosociales, esto repercutirá de forma directa en niveles de riesgo de estudiantes. Además, da a conocer que bastantes estudiantes presentan problemas de autoestima por diversos motivos, y si no saben desarrollar habilidades psicosociales tendrán problemas de adaptación.

Márquez (2015) afirma que las aptitudes son "arreglos mentales a través de los cuales el sujeto muestra concretamente la progresión del movimiento con el objetivo de crear, cambiar, hacer objetos, abordar problemas y seguir a uno mismo" (p.101). Por lo tanto, se alude al límite y la conducta hacia alguna actividad.

Para Serafino (2012) el término aptitud "se origina en el modelo mental de ajuste de conducta y se utiliza para expresar que la habilidad social no es una característica de identidad, sino una disposición de prácticas educadas y adquiridas" (p.76).

En cuanto a lo psicosocial El avance psicosocial de UNICEF (2004) es un procedimiento de cambio organizado y gradual en el que, en colaboración con la tierra, se logran niveles progresivamente complejos de desarrollos y actividades, de pensamiento, dialecto, sentimientos, emociones, y de asociaciones con otros.

\section{Habilidades psicosociales}


Como lo señala el MINSA (2013) llama la atención que las aptitudes sociales son la capacidad del individuo para desarrollar una conducta que persigue los determinantes del bienestar, predominantemente en las formas de vida. Es importante lograr que el inmaduro conozca y tenga sus capacidades y aptitudes específicas que, cuando se utilizan como parte de su día a día, le permitan tener formas de vida más ventajosas.

Un destacado entre los significados más reconocidos de aptitudes sociales es la dada por Caballo (2010), referido por Escales y Pujantell (2014) que establece: aptitudes sociales son un arreglo de prácticas producidos por la persona en un entorno relacional, que comunica sus emociones, mentalidades, deseos, sentimientos o derechos, de una manera que es propia de las circunstancias.

Para Muñoz y Crespi (2011) las aptitudes sociales aluden a aquellas particulares e importantes para conectarse e identificarse con el otro de manera convincente, aceptable y socialmente efectiva. Goleman (1999) en cuanto a las habilidades sociales, expresa que la comprensión de los demás debe ser subrayada, la introducción hacia el beneficio, el avance de los demás, la utilización de variedad y la atención política.

Bajo este contexto, esta investigación servirá para conocer la realidad tal cual como se presenta y poder ser material base para posibles programas de intervención. Tiene una relevancia teórica, pues la teoría indica que son diversos los factores de riesgo a los que están expuestos los estudiantes y que en la práctica se podrá corroborar. Con esta investigación no solo se beneficiarán los estudiantes, sino también los docentes quienes podrán asumir medidas preventivas y de intervención; al igual que los padres de familia, podrán ser parte de este cambio conociendo la realidad de sus hijos.

Por lo mencionado esta investigación tiene una proyección social ya que no solo abarcará la realidad palpable dentro de la institución sino también en el contexto familiar. Solo si conocemos la realidad actual podremos resolver en alguna medida el problema localizado. Siendo trascendental esta etapa de la 
vida para el desarrollo integral del estudiante que necesita ser evaluado y conocer las implicancias que este puede llevar dentro de su desempeño educativo.

Se planteó como objetivo determinar la influencia de las habilidades psicosociales en el nivel de riesgo de estudiantes de IIEE del nivel Secundario del distrito de Hunter 2018 y su hipótesis fue Hi: Existe influencia de las habilidades psicosociales en el nivel de riesgo de los estudiantes de IIEE del nivel Secundario del distrito de Hunter 2018.

\section{Principales habilidades psicosociales}

a. Información de uno mismo: infiere percibir nuestro ser, carácter, cualidades, deficiencias y preferencias.

b. Empatía: es simplemente la capacidad de "ponerse en los zapatos de los demás" y visualizar lo que la vida se asemeja a ese individuo

c. Correspondencia convincente o confiada: tiene que ver con la capacidad de transmitir lo que necesita ser, tanto verbal como preverbal, de una manera adecuada a la cultura y las circunstancias.

d. Conexiones relacionales: es la aptitud que nos hace relacionarnos enfáticamente con la población en general con la que colaboramos.

e. Liderazgo básico: nos simplifica el manejo útil de las opciones en lo que respecta a nuestras vidas y la de los demás.

f. Disposición de los problemas y los enfrentamientos: nos permite ver productivamente los problemas a lo largo de la vida cotidiana.

g. Razonamiento imaginativo: Consiste en la utilización de puntos de vista esenciales para crear o desarrollar pensamientos o elementos nuevos

h. Razonamiento básico: la capacidad de desglosar los datos y los encuentros en una forma de objetivo, aumenta el bienestar y el progreso individual y social

I. Supervisión de sentimientos y sentimientos: nos anima a percibir nuestros sentimientos y los de los demás para saber cómo afectan nuestra conducta social 
j. Administración de estrés: nos hace percibir las fuentes del estrés y sus impactos en nuestras vidas

\section{Nivel de riesgo psicosociales en los adolescentes}

La adolescencia al ser un período básico sujeto a cambios constantes en la mejora física, mental y social del menor, y en el entorno familiar en el que se crea, se considera como un momento de riesgo en el que se pueden dar las bases para la presencia de efectos secundarios e infecciones, y ajustes adicionales de la identidad.

El pensamiento crucial de este enfoque es que el peligro nos proporciona una medida del requisito de seguro social. El aprendizaje del peligro o la probabilidad de problemas médicos futuros pueden prever una atención suficiente y conveniente.

\section{Teoría de la Habilidad Social.}

Combs y Slaby (1977) citado por Milicic, (2010) la definen como la capacidad que posee el individuo para interactuar con los demás dentro de un contexto social. Tal y como lo afirma Libed y Lewinsohn (1983) citado por Milicic (2010) reforzará positiva o negativamente las conductas desarrolladas, generando que éstas continúen o se extingan. Esto nos muestra que las habilidades sociales ya no solo tienen relación con los que nos rodean, sino que un factor determinante en esto será el contexto en que estas se van a desarrollar y que será el que determine la adecuación de las mismas.

Esta capacidad normal de información relacional ha sido durante bastante tiempo, la medida central de la visión social. Hay teóricos que confiesan que la percepción social, en cuanto a la visión general conectada al mundo social, constituye la principal medida del conocimiento social. Sea como sea, esta visión se centra en lo que conocemos del mundo relacional que en el modelo genuino en el que nos identificamos con los demás, lo que ha provocado medidas de percepción social que, a pesar de que demuestran nuestra comprensión de las circunstancias sociales, pasan por alto la manera en que nos movemos en ellas.

\section{Teoría del aprendizaje sociocultural}


Lev (1979) citado por Milicic, M. (2010) sostiene que el aprendizaje mueve una progresión de formas de mejora interior que solo comienza cuando el niño se conecta con las personas en su condición y con sus compañeros. El aprendizaje se considera social y lógicamente particular, donde la partición de la persona de sus impactos sociales es inconcebible. Mientras tanto, la condición social es fundamental para el aprendizaje.

Cada capacidad en el progreso social del niño aparece en dos planos: primero en el plano social y luego en el plano mental; de esta manera, la mejora intelectual requiere la cooperación social con personas alternativas de la reunión. Vygotsky (1979) citado por Milicic, M. (2010) sostiene que los nuevos niveles de información comienzan en un nivel relacional: inicialmente entre el joven y el adulto y luego a través de una conexión social constante. Porque la incorporación de elementos sociales y mentales potencia y fortalece el aprendizaje. Por lo tanto, podemos declarar que el tema es famoso socialmente y la información es, además, un elemento social.

\section{Teoría del aprendizaje social}

Bandura, citado por Solano (2015) afirma que una conducta decente, grande y horrible, típica e irregular se descubre al emular la conducta de otros individuos. Desde la juventud, creamos reacciones a los modelos que la sociedad nos ofrece. Con los guardianes como los modelos principales, asimilamos su dialecto y lo asociamos con las tradiciones y prácticas adecuadas de la forma de vida. Las personas que van mal de las normas sociales han adoptado su conducta de manera similar a cualquier otra persona. La distinción es que las personas anormales han tomado después de los modelos que lo que queda de la sociedad piensa desafortunado.

Bandura es un crítico del tipo de sociedad que ofrece a sus hijos modelos incorrectos, especialmente los casos de conducta feroz, que son el estándar en TV, películas y juegos de computadora. Su exploración obviamente demuestra el impacto de los modelos en la conducta. En el caso de que avancemos para convertirnos en lo que vemos, en ese momento la separación entre echar un vistazo a un personaje enérgico y realizar actos viciosos no es excesivamente increíble. 
Las cualidades de los modelos influyen en nuestra inclinación a copiarlos. Podemos obtener más impacto de alguien que nos parece que de alguien que contrasta con nosotros de manera clara y significativa. Es probable que demostremos nuestra conducta con la de un hombre de sexo indistinguible con la de la inversa. Además, probablemente nos veamos afectados por los modelos de nuestra edad. Los conjuntos que parecen haber abordado eficazmente los problemas que enfrentamos son modelos extremadamente persuasivos.

\section{Teoría para la conducta de riesgo de los adolescentes de Jessor}

Una definición que Jessor (1991) citado por Milicic, M. (2010) da de la conducta peligrosa es que es "cualquier conducta que puede cambiar las partes psicosociales de la mejora fructífera de la juventud" (p.599). Mientras tanto, exige que utilice el grupo de conducta peligrosa y no el de peligro buscando conducta, con el argumento de que el último término lo considera penoso, ya que es repetitivo aquí y allí e inspira la convicción de que todos los jóvenes son buscadores de peligro. Sea como sea, para él este grupo sería adecuado para aquellos adolescentes que conocen el peligro y además lo buscan a propósito.

En esta línea, confía en que en los adolescentes es más inteligente utilizar la categoría de conducta peligrosa que la posibilidad de perseguir, con el argumento de que, además, un gran número de prácticas incluirán el azar, por ejemplo, probar sustancias, participar en relaciones sexuales, conducir, conferir actos brutales, etc. Se considera que el grupo de conducta peligrosa puede estar conectado a cualquier conducta que se elija con el avance del joven. Esto lleva, en ese punto, a un sueño en un nivel teórico más amplio.

\section{MÉTODO}

Su investigación es descriptiva - correlacional, porque se trata de establecer la a relaciones que pudiera haber entre las variables. Es transversal porque 
los datos a recogerse se darán en un solo momento, en un solo tiempo. Es correlacional, por qué busca establecer correlaciones entre las variables habilidades psicosociales y nivel de riesgo, para determinar la influencia en un grupo de personas (Hernández y Baptista, 2003).

La población, objeto de estudio; es censal constituida por todos los estudiantes de quinto de secundaria de las dos Instituciones Educativas del nivel secundario, sector público del distrito de Jacobo Hunter de Arequipa que son en número de 265.

Tabla 1: Población y muestra de estudiantes por instituciones Educativas

\begin{tabular}{|c|c|c|}
\hline Instituciones educativas & Nro de estudiantes & $100 \%$ \\
\hline Juan Pablo Vizcardo y Guzmán & 120 & $45.28 \%$ \\
\hline San Antonio María Claret CIRCA & 145 & $54.72 \%$ \\
\hline Total & 265 & $100 \%$ \\
\hline
\end{tabular}

Fuente: elaboración propia

La encuesta, que según Arias (1999), es un procedimiento que se utiliza con el propósito conseguir información importante definida por cualidades o características preponderantes de una población o unidades de estudio que consiste en interrogar, y consolidar las respuestas como información registrada.

Los instrumentos para la variable 1: habilidades psicosociales es la escala de habilidades social y para la variable y su confiabilidad fue de 0.908 la cual es alta según la escala de alfa de Crombach, 2: niveles de riesgo es el cuestionario para medir los factores de riesgo y su confiabilidad fue de 0.844 la cual es alta según la escala de alfa de Crombach.

\section{RESULTADOS}

Los resultados encontrados manifiestan el análisis de las siguientes variables. 
Tabla 2: Variable 1. Habilidades Psicosociales de los estudiantes de IIEE del nivel Secundario del distrito de Hunter.

\begin{tabular}{lll}
\hline Nivel & Frecuencia & Porcentaje \\
\hline Alto & 150 & 56,60 \\
Regular & 35 & 13,20 \\
Bajo & 80 & 30,20 \\
\hline TOTAL & $\mathbf{2 6 5}$ & $\mathbf{1 0 0 , 0 0}$ \\
\hline
\end{tabular}

Fuente: Registro de datos Anexo

La Tabla 1, referido a la variable 1: habilidades psicosociales, muestra que un $56.60 \%$ se ubica en el nivel alto. En seguida con un $30.20 \%$ se ubica en el nivel bajo y con $13.2 \%$ en el nivel regular.

Tabla 3: Variable 2: Nivel de riesgo de los estudiantes de IIEE del nivel Secundario del distrito de Hunter.

\begin{tabular}{lll}
\hline Nivel & Frecuencia & Porcentaje \\
\hline Alto & 80 & 30,20 \\
Regular & 91 & 34,30 \\
Bajo & 94 & 35,50 \\
\hline TOTAL & $\mathbf{2 6 5}$ & $\mathbf{1 0 0 , 0 0}$ \\
\hline
\end{tabular}

Fuente: Registro de datos Anexo

La tabla 2, referido a la variable 2: nivel de riesgo, muestra que un $35.50 \%$ se ubica en el nivel bajo, en seguida con un $34.30 \%$ se ubica en el nivel regular y un $30.20 \%$ en el nivel alto.

Existe influencia de las habilidades psicosociales en el nivel de riesgo de los estudiantes de IIEE del nivel Secundario Distrito de Hunter 2018.

Figura 1: Diagrama de dispersion entre las habilidades psicosociales y nivel de riesgo 


\section{Diagrama de dispersion entre las habilidades psicosociales y nivel de riesgo}

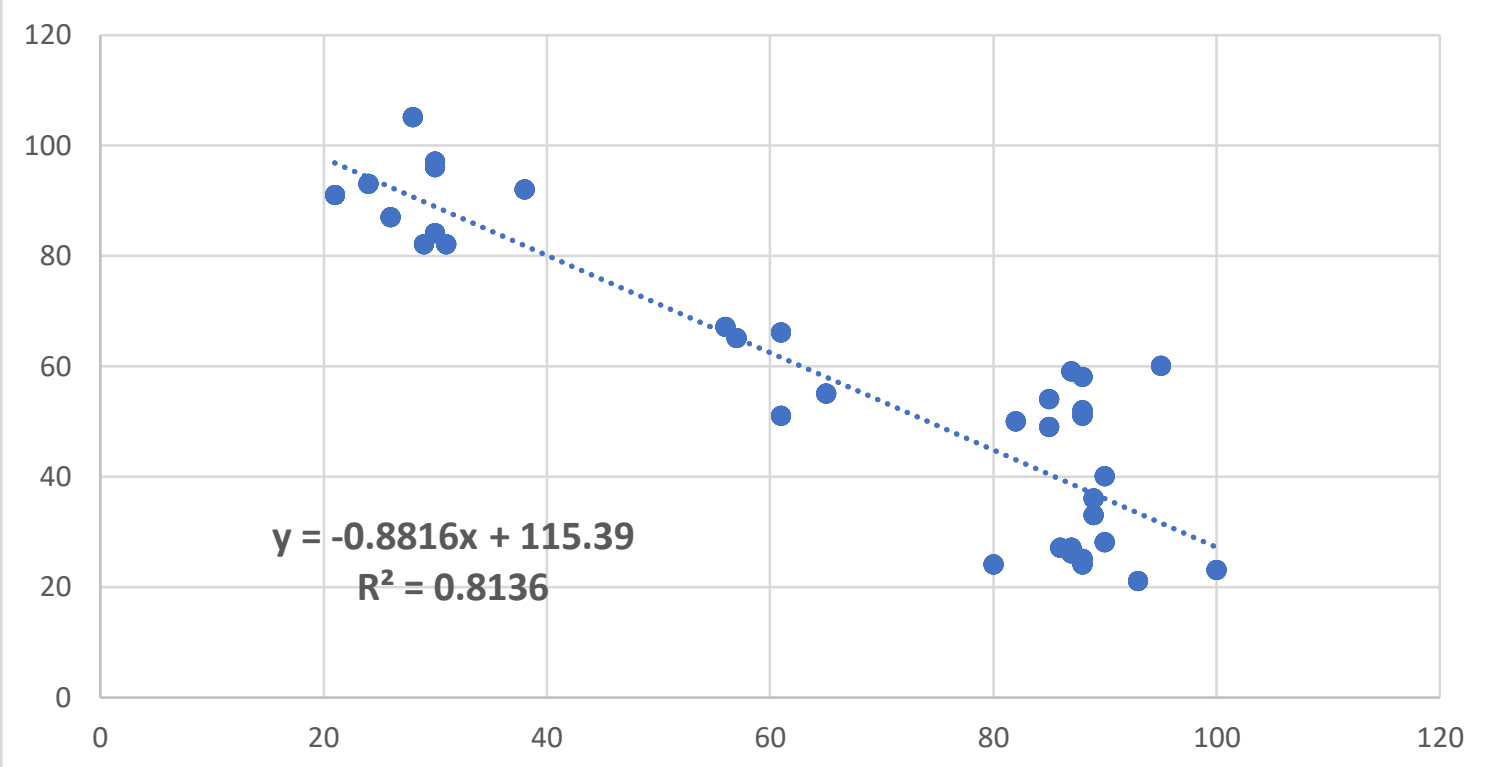

Fuente: elaboración propia

Se observa los puntos de dispersión que grafica la correlación entre las dos variables de estudio de las habilidades psicosociales y nivel de riesgo. Se evidencia una distribución positiva, la dispersión de puntos está cerca a la recta de regresión lineal, lo cual afirma que existe correlación entre las variables.

\section{DISCUSIONES}

La presente investigación tiene la finalidad determinar la influencia de las habilidades psicosociales en el nivel de riesgo de estudiantes de IIEE del nivel Secundario distrito de Hunter 2018. Para tal fin se aplicaron los instrumentos para cada variable. Asimismo, se realizó la estadística descriptiva con sus tablas y gráficos. En la estadística inferencial se realizó la correlación regresión lineal para cada variable y sus respectivas dimensiones. Dentro de ello se realizó las concordancias de los antecedentes de investigación.

Es importante, determinar la influencia de las habilidades psicosociales en el nivel de riesgo de estudiantes de IIEE del nivel secundario distrito de Hunter 2018. Es un tema que favorece al desarrollo de habilidades psicosociales del estudiante y abarca aspectos relacionados a los niveles riesgo individual, familiar y social. 
En cuanto a los resultados de la comprobación de hipótesis, se aplicó la prueba estadística correlación regresión lineal para establecer la correlación entre las variables habilidades psicosociales y niveles de riesgo. Se obtiene como valor de correlación 0,814 , lo que determina una correlación negativa alta entre ellas, con un nivel de significancia de 0,000 menor a 0,05 nos permite validar la hipótesis de investigación y rechazar la hipótesis nula indicando que las habilidades psicosociales influyen en el nivel de riesgo de los estudiantes de IIEE del nivel secundario distrito de Hunter 2018.

El primer objetivo pide determinar el nivel de habilidades psicosociales de los estudiantes de IIEE del nivel secundario distrito de Hunter. Según los resultados $56.60 \%$ se ubica en el nivel alto, en seguida con un $30.20 \%$ se ubica en el nivel malo y con $13.2 \%$ en el nivel regular.

El segundo objetivo pide identificar el nivel de riesgo de los estudiantes de IIEE del nivel secundario distrito de Hunter, según los resultados muestra que un $35.50 \%$ se ubica en el nivel malo, en seguida con un $34.30 \%$ se ubica en el nivel regular y es un $30.20 \%$ en el nivel alto.

El tercer objetivo busca determinar el grado de relación entre las habilidades psicosociales y el nivel de riesgo individual de los estudiantes IIEE del nivel secundario distrito de Hunter. En los resultados, la regresión lineal entre las habilidades psicosociales y su dimensión nivel de riesgo individual es 0.771 , lo que indica que existe una correlación negativa alta entre la variable y dimensión. Es decir, a mayor habilidad psicosocial mayor nivel de riesgo individual de los estudiantes IIEE del nivel Secundario Distrito de Hunter.

En el cuarto objetivo pide determinar el grado de relación entre las habilidades psicosociales y el nivel de riesgo familiar de los estudiantes IIEE del nivel secundario distrito de Hunter. La correlación entre las habilidades psicosociales y su dimensión nivel de riesgo familiar es 0,753. Es decir, a mayor habilidad psicosocial, mayor nivel de riesgo familiar de los estudiantes IIEE del nivel secundario distrito de Hunter. 
Finalmente, el quinto objetivo pide determinar el grado de relación entre las habilidades psicosociales y el nivel de riesgo social de los estudiantes IIEE del nivel secundario distrito de Hunter. La correlación regresión lineal entre las habilidades psicosociales y su dimensión nivel de riesgo social es 0,797.

Los resultados coinciden con lo hallado por Florez, (2016) en su tesis de maestría titulada: Aplicación de un programa de habilidades psicosociales basado en el autoconocimiento para fortalecer las relaciones interpersonales de los niños y niñas de tercer grado de primaria de lEP Marvista Paita, 2013. Donde se demostró que los estudiantes tienen deficientes relaciones interpersonales o son conflictivos, teniendo en cuenta que falta de autoconocimiento de los estudiantes como también deficiente comunicación que origina los conflictos en la socialización.

También se concuerda con Silva, (2015) quien demostró un alto nivel de cumplimiento con la puesta en marcha del programa de intervención por parte de educadores y estudiantes. Así como la visión subjetiva de los estudiantes después de su sección a través del programa.

\section{CONCLUSIONES}

En base a los datos recogidos en la investigación se ha logrado determinar un coeficiente de correlación regresión lineal de $r=0,814$ que indica que existe influencia de las habilidades psicosociales en el nivel de riesgo de los estudiantes de IIEE del nivel Secundario Distrito de Hunter 2018.

Se ha logrado determinar el nivel de habilidades psicosociales, que 56.60\% se ubica en el nivel alto, en seguida con un $30.20 \%$ se ubica en el nivel malo y con un $13.2 \%$ en el nivel regular, lo cual indica, que el nivel de habilidades psicosociales influye significativamente en los estudiantes de IIEE del nivel secundario distrito de Hunter. 
El nivel de riesgo de los estudiantes en un $35.50 \%$ se ubica en el nivel malo, en seguida con un $34.30 \%$ se ubica en el nivel regular y es un $30.20 \%$ en el nivel alto. Lo cual indica, que los niveles de riesgo influyen significativamente en los estudiantes de IIEE del nivel secundario distrito de Hunter.

Se ha logrado determinar un coeficiente de correlación regresión lineal de r= 0,771 que existe influencia de las habilidades psicosociales en el nivel de riesgo individual de los estudiantes IIEE del nivel secundario distrito de Hunter.

Se ha logrado determinar un coeficiente de correlación regresión lineal de r= -0,753 que existe influencia de las habilidades psicosociales en el nivel de riesgo familiar de los estudiantes IIEE del nivel secundario distrito de Hunter.

Se ha logrado determinar un coeficiente de correlación regresión lineal de $\mathrm{r}=$ $-0,797$ que existe influencia de las habilidades psicosociales en el nivel de riesgo social de los estudiantes IIEE del nivel secundario distrito de Hunter.

\section{REFERENCIAS}

Arias, F. (2010). El proyecto de investigación: Introducción a la metodología científica (6a ed.). Caracas: Episteme.

Bermejo, L. y Fernández, G. (2017). Habilidades sociales y resolución de conflictos en centros docentes de Andalucía. Universidad de Sevilla España, para optar el grado de Magister.

Caballo, V. (2010), Teoría, evaluación y entrenamiento de las habilidades sociales. Valencia: Promolibro.

Escales, R., y Pujantell, M. (2014). Habilidades sociales. Madrid: Macmillan 
Iberia, S.A.

Flórez, M. (2016), Aplicación de un programa de habilidades psicosociales basado en el autoconocimiento para fortalecer las relaciones interpersonales de los niños y niñas de tercer grado de primaria de lEP Marvista Paita, 2015. Universidad Nacional San Agustín.

Goleman, D. (1999). Inteligencia Social. Barcelona: Editorial Kairos.

Hernández, R.; Fernández, C. y Baptista, P. (2010). Metodología de la Investigación. México: Mc Graw-Hill.

Márquez, A. (2015). Las habilidades, reflexiones y proposiciones para su evaluación. En: Aleida Márquez, Clara Suárez Rodríguez. Manual de consulta para la maestría en Ciencias Pedagógicas. Santiago de Cuba: Universidad de Oriente.

Milicic, M. (2010). Vivir con otros. Santiago de Chile: Universitaria.

MINSA (2013) Manual de habilidades sociales en adolescentes escolares. Ministerio de Salud. Dirección General de Promoción de la Salud. Dirección Ejecutiva de Educación para la Salud; Instituto Especializado de Salud Mental "Honorio Delgado/Hideyo Noguchi”. Catalogación hechapor el Centro de Documentación OPS/OMS en el Perú Lima.

Muñoz, H., y Crespi, J. (2011). Enseñanza de la Psicología Social en la Formación Continua de los profesionales de la salud: orientaciones prácticas desde un Modelo de Competencia Social. Valencia: NAU Libres

Serafino, P. (2012). Health Psichology, Biopschosocial interactions. U.S.A. University Of United States of America.

Silva, L. (2015). La mediación como herramienta para resolver conflictos. Impacto 
sobre las habilidades sociales de los alumnos mediadores en uncentro de educación secundaria. Para la obtención del grado de doctor en educación en la Universidad de Alcalá - España.

Solano, L. (2015). Rendimiento académico de los estudiantes de secundaria obligatoria y su relación con las aptitudes mentales y las actitudes anteel estudio. (Tesis de doctorado). Recuperado de: http://espacio.uned.es/fez/eserv/tesisuned:EducacionLosolano/SOLANO_ LUENGO_Luis_Octavio.pdf. (9/10/2016).

UNICEF (2004) Informe Anual. Fondo de la Naciones unidades para la infancia.

New York. USA.

Verde, R. (2015) Taller aprendiendo a convivir para el desarrollo de habilidades sociales en los alumnos del primer año de educación secundaria de la I.E. Víctor Raúl Haya de la Torre. El Porvenir - Trujillo, 2016. Tesis de magister, Universidad Cesar Vallejo - Perú. 